\title{
10 Jahre danach
}

\author{
Helmut Tschiersky-Schöneburg
}

Online publiziert: 5 Oktober 2012

(C) Bundesamt für Verbraucherschutz und Lebensmittelsicherheit (BVL) 2012

In diesen Tagen feiern viele Lebensmittelbehörden in Deutschland und Europa ihr zehnjähriges Bestehen: Die sogenannte "BSE-Affäre" hatte am Ende des vergangenen Jahrtausends zu einem bis dahin nicht gekannten Vertrauensverlust der Verbraucher in das System der Lebensmittelüberwachung geführt. Als wichtige Konsequenz aus den Geschehnissen wurde mehr Koordination und mehr Transparenz in den Entscheidungen der verantwortlichen Stellen gefordert.

\section{Vom Acker bis zum Teller}

Ein von der Europäischen Kommission in Auftrag gegebenes und im Jahr 2000 erschienenes "Weißbuch zur Lebensmittelsicherheit" hob neben der Empfehlung der Einrichtung einer unabhängigen Europäischen Lebensmittelbehörde, der späteren "European Food Safety Agency (EFSA)", die Notwendigkeit hervor, das Kontrollsystem von Grund auf zu reformieren und einen neuen gemeinschaftsrechtlichen Rahmen zu bilden. Mit der sogenannten “Basisverordnung” VO(EG) Nr. 178/2002 wurde dieser Rechtsrahmen geschaffen und gleichzeitig wichtige Grundprinzipien des neuen Systems festgeschrieben. Seine Grundlage bildet die Wertschöpfungskette, die ein Lebensmittelerzeugnis als Produkt aufeinanderfolgender Prozessstufen "vom Acker bis zum Teller” begreift. Die primäre Verantwortung für die

Dr. H. Tschiersky-Schöneburg ( $\square)$

Bundesamt für Verbraucherschutz und

Lebensmittelsicherheit, Mauerstr. 39-42,

10117 Berlin, Germany

e-mail: helmut.tschiersky-schoeneburg@bvl.bund.de
Sicherheit des Lebensmittels liegt beim Lebensmittelunternehmer, der in seinem Verantwortungsbereich ein Eigenkontrollsystem etablieren muss und damit auch ein Risikomanagement betreibt. Auf staatlicher Seite soll eine klare Trennung zwischen einer unabhängigen wissenschaftlichen Risikobewertung und dem Risikomanagement erfolgen. Rückverfolgbarkeit in der Wertschöpfungskette und Risikoorientierung der staatlichen Überwachung, die sich primär als “Kontrolle der Kontrolle” versteht, sowie das europaweit angelegte behördliche Schnellwarnsystem RASFF sind weitere Grundsätze dieses neuen europäischen Rechtsrahmens.

Die spannende Frage war nun, wie diese Vorgaben im föderalen System der Bundesrepublik Deutschland umgesetzt werden sollen. Die Überwachung des Verkehrs mit Lebensmitteln liegt in der Zuständigkeit der Bundesländer, die ihrerseits diese Aufgabe auf weit mehr als 400 kommunale Ämter übertragen haben. Durch die enge Verzahnung der nationalen mit der europäischen Ebene ergibt sich aber die Notwendigkeit gesamtstaatlichen Handelns, das eine bundesweite Koordination der Überwachungstätigkeit und damit des Risikomanagements voraussetzt.

Das von der Bundesregierung in Auftrag gegebene Gutachten der Präsidentin des Bundesrechnungshofes, Hedda von Wedel, führte zur Gründung des Bundesamtes für Verbraucherschutz und Lebensmittelsicherheit (BVL) und des Bundesinstituts für Risikobewertung (BfR) im Jahre 2002.

Zu diesem Zeitpunkt wurde die Neuorganisation des gesundheitlichen Verbraucherschutzes auf nationaler Ebene im Sinne bundeseinheitlicher Vorgaben vorangetrieben. Damit wollte man die Handlungsspielräume, die die europa- und unionsrechtlichen Vorgaben 
eröffneten, ausnutzen. Das BVL sollte die Diskussion bei der Entwicklung allgemeiner Verwaltungsvorschriften in zwei Bund-Länder-Ausschüssen koordinieren. Diese Ausschüsse haben die Aufgabe aber nie wahrnehmen können. Sollte es ein Ziel der Neuordnung gewesen sein, die damals existierenden Bund-Länder-Gremien zugunsten einer klaren Organisationsstruktur unter Vorsitz des Bundes abzulösen, so muss dieses Vorhaben aus heutiger Sicht als nicht erreicht betrachtet werden. $\mathrm{Zu}$ diesem Schluss kommt der Bundesbeauftragte für Wirtschaftlichkeit in der Verwaltung in seinem Gutachten „Organisation des gesundheitlichen Verbraucherschutzes (Schwerpunkt Lebensmittel) “ vom Oktober 2011.

Das BVL entwickelte in den Folgejahren seine Aufgaben im Bereich des Risiko- und Krisenmanagement sowie beim zentralen Datenmanagement fort und traf die organisatorischen Vorbereitungen für den Fall einer Krise. Ein Lagezentrum, in dem alle für die Erstellung eines Lagebildes notwendigen Informationen zusammenlaufen, unterstützt im Fall einer Krise das Bundesministerium. Da das Risikomanagement entlang der gesamten Wertschöpfungskette betrieben wird, erhielt das Bundesamt bei seiner Gründung die Zuständigkeiten für die Zulassung von Pflanzenschutzmitteln und Tierarzneimitteln sowie ab 2004 für gentechnisch veränderte Organismen. Acht nationale und ein europäisches Referenzlabor runden das Management-Instrumentarium des BVL im gesundheitlichen Verbraucherschutz ab.

Die ersten Jahre seines Bestehens waren somit vom Aufbau und einer Standortbestimmung des Bundesamtes im neu geschaffenen System des gesundheitlichen Verbraucherschutzes bestimmt. Heute versteht es sich vor allem als Dienstleister und Partner der Länder und europäischen Institutionen sowie aller anderen wichtigen Stakeholder aus den Bereichen Verbraucherschutz, Wirtschaft, Wissenschaft und Politik. Seit 2006 nimmt das BVL auch Aufgaben im wirtschaftlichen Verbraucherschutz wahr, was den Rahmen seines Tätigkeitsfeldes weiter öffnet.

\section{Quo vadis, BVL?}

Das weite Feld des Verbraucherschutzes ist durch sich ständig wandelnde Anforderungen geprägt. Dabei gaben besonders die Ereignisse des Jahres 2011, wie die EHEC-Krise, der Entwicklung neuer Managementinstrumente einen spürbaren Schub. Erstmals kamen in einer Task Force die im operativen
Krisenmanagement eingebundenen Stellen aus dem Bund (BVL, BfR, RKI), der betroffenen Bundesländer sowie der EFSA in der Berliner Dienststelle des BVL zusammen. Sie leisteten gemeinsam die zum Krisenmanagement notwendige Aufklärungsarbeit. Dieses Modell wird nun institutionalisiert und für künftige vergleichbare Lagen schnell verfügbar gemacht. Darüber hinaus soll es künftig eine elektronische Datenbank geben, in die die Bundesländer ihre Überwachungsdaten einpflegen und damit zur Früherkennung von Risiken und für die Erstellung von Lagebildern nutzbar machen.

Auch außerhalb der Krise entwickelt das BVL den Verbraucherschutz fort: Globale Warenströme machen das Angebot von Lebensmitteln auf unserem Speisetisch vielfältiger. Sie bringen aber auch die Risiken der Ursprungsländer zu uns. Das BVL engagiert sich deshalb verstärkt in Drittländern durch die Teilnahme an Twinning- und TAIEX-Maßnahmen, Projekten oder bilateralen Kooperationen. So reicht heute die Landkarte des BVL von Marokko über Moldau bis nach China.

Die Globalisierung ist im Internet am weitesten fortgeschritten; hier liegen Chancen und Risiken dicht beieinander. Auch Lebensmittel werden über diesen Vertriebsweg gehandelt, und nicht selten sind angeblich gut verträgliche, „rein natürliche“ Erzeugnisse nicht zugelassene Novel Food oder gar Arzneimittel mit stark wirksamen Arzneistoffen. Sowohl eine Zentralstelle zur Überprüfung des Internethandels als auch eine gemeinsame Expertenkommission mit dem Bundesinstitut für Arzneimittel und Medizinprodukte (BfArM) beim BVL werden sich zukünftig dieser besonderen Thematik des gesundheitlichen Verbraucherschutzes annehmen.

Im wirtschaftlichen Verbraucherschutz möchte das BVL Entwicklungspotentiale nutzen, um die in Deutschland traditionell durch Verbände wahrgenommene Rechtsdurchsetzung über den bisherigen Rahmen hinaus effektiv zu erweitern.

\section{Transparenz schafft Vertrauen}

Die Information des Verbrauchers wird wie bisher auch in Zukunft eine Schwerpunktaufgabe des BVL bilden. Dabei werden Portalanwendungen eine wichtige Rolle spielen, wie das gemeinsam mit den Bundesländern betriebene Portal http://www. lebensmittelwarnung.de.

Ein Zeitgenosse merkte einmal an, die beste Behörde sei die, über die man wenig spricht. Tatsächlich hat das BVL in seiner noch jungen 
Geschichte zahlreiche Prüfungen erfolgreich bestanden, ohne dass die Öffentlichkeit davon im nennenswerten Maße Notiz genommen hat.

Der Erfolg dieser Behörde ist vor allem dem Engagement und der Qualifikation ihrer Mitarbeiterinnen und Mitarbeiter geschuldet, die sich trotz vieler Schwierigkeiten nie entmutigen ließen.
Eine lebendige Behörde muss sich auch in ihrer Struktur den sich wandelnden Anforderungen anpassen. Deshalb wird sich das Bundesamt in den nächsten Jahren so aufstellen, dass es den künftigen Aufgaben gewachsen ist. Denn im Mittelpunkt steht der Verbraucher, ganz im Sinne des BVL-Claims „Risiken managen - Verbraucher schützen.“ 